Rev. Elev. Méd. vét. Pays trop., 1977, 30 (2) : 175-180.

\title{
Pâturages de la zone tropicale humide. Connaissances acquises et besoins en recherches complémentaires
}

\author{
par G. BOUDET (*)
}

\begin{abstract}
RÉSUMÉ
En zone tropicale humide, les connaissances sur les formations végétales et leur productivité primaire nette permettent de formuler des principes fondamentaux de gestion des pâturages naturels. Cependant les écosystèmes pâturés ne se conçoivent qu'en y integrant l'animal herbivore et il est urgent de préciser leur production secondaire évaluée en viande, car les données fournies par les exploitations existantes sont souvent insuffisantes ou trop fragmentaires.
\end{abstract}

A l'exception des régions d'altitude, la zone tropicale humide n'est pas traditionnellement une terre d'éleveurs et la recherche sur les pâturages tropicaux humides doit en tenir compte et présenter l'originalité d'une approche intégrant l'estimation des ressources pastorales dans un contexte où spéculation agricole et spéculation élevage ne peuvent être qu'étroitement imbriquées.

\section{RESSOURCES PASTORALES EN ZONE TROPICALE HUMIDE}

La zone tropicale humide est un terme ambigu et les limites peuvent varier selon les auteurs. Pour les pâturages, il semble judicieux de considérer la région comprise entre l'équateur et la limite d'extension des forêts claires, forêts dont les arbres ont des cimes plus ou moins jointives. Cette zone englobe donc la forêt dense sempervirente équatoriale, les forêts denses sèches où seul le sous-bois est sempervirent, les forêts

(*) Directeur de Recherches agropastorales. I. E. M. V. T., 10 , rue Pierre-Curie, 94700 MaisonsAlfort, France. claires où des graminées peuvent subsister dans le sous-bois et les différents types de savanes, plus ou moins boisées constituant des faciès de substitution des forêts primitives à la suite des défrichements, des mises en cultures et des feux répétés.

Les conditions climatiques varient du climat équatorial au climat tropical humide. Au voisinage de l'équateur, secteur de la forêt dense équatoriale, la pluviosité moyenne annuelle est supérieure à $1600 \mathrm{~mm}$ avec un régime de deux saisons des pluies, les saisons sèches intercalaires se traduisant surtout par une diminution relative des pluies et la période active $\left({ }^{*}\right)$ de la végétation herbacée y est toujours supérieure à 10 mois. Au-delà, les conditions d'aridité augmentent avec possibilité de distinguer 3 secteurs :

- le secteur périforestier délimité par une

(*) La période active est la période pendant laquelle les besoins d'eau pour l'évaporation, phénomène physique dépendant du degré hygrométrıque de l'air et pour la transpiration des plantes, phénomène physiologique, sont comblés par les précipitations (pluies, rosée) ou par les réserves en eau accumulées dans les horizons du sol prospectables par les racines. 
période active supérieure à 7 mois et une pluviosité annuelle de 1300 à $1500 \mathrm{~mm}$;

- le secteur soudano-guinéen délımité par une période active supérieure à 5 mois et une pluviosité annuelle supérieure à $1000-1300 \mathrm{~mm}$ avec pour limite nord: Casamance, sud Mali, sud Haute-Volta, nord Ghana, centre Nigeria, nord Adamaoua.

Parallèlement, avec l'altitude qui atténue les caractères tropicaux du climat, peuvent être distingués :

- le secteur des plateaux entre 800 et $1500 \mathrm{~m}$ d'altitude ;

- le secteur altimontain, au-dessus de $1500 \mathrm{~m}$ d'altitude.

La production pastorale dépend de la longueur de la période active, du potentiel de fertilité des sols qui varie avec la nature géologique du sous-sol, le modelé du terrain, la situation topographique, et surtout du rapport d'équilibre existant entre strate herbacée et strate ligneuse, cette dernière tendant à dominer dès que les herbes sont consommées par du bétail.

En secteur forestier, des savanes sur terrain sableux à Brachiaria brachylopha et Hyparrhenia diplandra peuvent fournir $3500 \mathrm{~kg} / \mathrm{ha}$ de MS appétible $\left(^{*}\right)(185 \mathrm{j} / \mathrm{an}$ de pâture d'un UBT).

En secteur périforestier, les savanes sur sol profond de plateaux à Hyparrhenia diplandra et Hyparrhenia rufa produisent 13 t/ha de MS (700 j/an de pâture d'un UBT). Sur terrains squelettiques gravillonnaires la savane à Loudetia arundinacea fournit $2500 \mathrm{~kg} / \mathrm{ha}$ de MS (130 j/an de pâture d'un UBT) de même que la savane à Andropogon ascinodis et Hyparrhenia smithiana sur terrains sablonneux de bas de pente.

En secteur soudano-guinéen, la production des savanes sur terrains pénéplanés à sol profond à Andropogon ascinodis et Diheteropogon amplectens atteint $3 \mathrm{t} / \mathrm{ha}$ de MS (160 j/an de pâture d'un UBT), celle des terrains squelettiques gravillonnaires à Loudetia simplex et Schizachyrium sanguineum, $2 \mathrm{t} /$ ha de MS (100 j/an de pâture d'un UBT), celle des terrains squelettiques cuirassés à Andropogon ascinodis et Loudetia

(*) La production herbacée (biomasse) des espèces pâturables est mesurée en fin de période active sur des plages enherbées, dépourvues de ligneux et permet d'estimer la charge théorique d'un pâturage débroussé sur la base d'un tiers de la production consommable par un UBT de $250 \mathrm{~kg}$ dont la consommation journalière serait de $6,25 \mathrm{~kg}$ de MS. simplex, 1,5 t/ha de MS ( $80 \mathrm{j} /$ an de pâture d'un UBT), celle des glacis colluviaux à Andropogon gayanus et Hyparrhenia smithiana, 3 t/ha de MS ( $160 \mathrm{j} /$ an de pâture d'un UBT) et celle des savanes des vallées inondables à Hyparrhenia rufa et Setaria sphacelata, $8 \mathrm{t} / \mathrm{ha}$ de MS $(420 \mathrm{j} / \mathrm{an}$ de pâture d'un UBT).

Sur les hauts plateaux, la production des savanes à Hyparrhenia rufa et Hyparrhenia diplandra sur terrains profonds est de $4 \mathrm{t} / \mathrm{ha}$ de MS ( $210 \mathrm{j} / \mathrm{an}$ de pâture d'un UBT) et celle des savanes à Loudetia arundinacea et Schizachyrium sanguineum est de 1,5 t/ha de MS ( $80 \mathrm{j} / \mathrm{an}$ de pâture d'un UBT).

En secteur altimontain, la production de la savane à Sporobolus africanus est également estimée à $1,5 \mathrm{t} / \mathrm{ha}$ de $\mathrm{MS}$ ( $80 \mathrm{j} / \mathrm{an}$ de pâture d'un UBT).

\section{EXPLOITATION DES PÂTURAGES EN ZONE TROPICALE HUMIDE}

A basse altitude, l'élevage bovin est traditionnellement une activité secondaire, le troupeau villageois constituant plutôt une forme d'épargne vivante. Les bovins sont des taurins trypano-résistants alors que les races zébus doivent être protégées par chimio-prévention. Les troupeaux pâturent sous gardiennage pendant la période des cultures, à l'extérieur des zones d'extension des terres cultivées. Après les récoltes, les troupeaux se déplacent librement et consomment d'une part les résidus de récoltes sur champs ainsi que les repousses d'adventices en particulier sur rizières, et d'autre part les repousses après feux, hors des terrains cultivés. La charge effective de saison sèche est alors estimée à 2 ha/UBT sur terres cultivées ou rizières et à $5 \mathrm{ha} / \mathrm{UBT}$ hors cultures après feux.

Avec l'augmentation des besoins en viande et l'extension de la culture attelée, cet élevage de cueillette doit faire place à une véritable spéculation élevage sur parcours extensifs ou prairies cultivées et quelques acquis peuvent être mentionnés, avec expérimentation sur taurillons.

En secteur soudano-guinéen du Nigeria (5) des parcours déboisés et brûlés en mars tous les 3 ans peuvent fournir :

- un gain de poids vif de $45 \mathrm{~kg} / \mathrm{ha}$ en saison des pluies, avec une charge d'1 ha/tête (45 kg/tête) ; 
- un gain de poids vif de $20 \mathrm{~kg} / \mathrm{ha} / \mathrm{an}$, avec une charge de $2,5 \mathrm{ha} /$ tête $(50 \mathrm{~kg} /$ tête/an).

Sur les plateaux du Cameroun (Wakwa), en saison des pluies, avec rotation et temps de repos de $25 \mathrm{j}$, on obtient un gain de poids vif de $140 \mathrm{~kg} / \mathrm{ha}$ avec une charge de $0,5 \mathrm{ha} /$ tête (70 kg/animal).

En saison sèche, avec une charge de 2 ha/tête, la perte peut atteindre 20 p. 100 par animal en fin de saison (50 kg/animal). Cette perte est annulée et remplacée par un gain de 5 p. 100 en distribuant une supplémentation d'un $\mathrm{kg}$ de tourteau de coton par tête pendant les 2 derniers mois de la saison sèche. Sans supplémentation et avec 2 ha/tête/an dont 0,5 ha pâturé en saison des pluies, le gain de poids par an n'est que de $10 \mathrm{~kg} / \mathrm{ha}$ (20 kg/animal).

Des résultats y ont été obtenus sur les risques d'embroussaillement et les moyens d'y remédier : avec pâture répétée en saison des pluies et suppression des feux, le pâturage évolue vers une forêt basse en 15 ans. Avec des feux pratiqués après mise hors pâture d'une saison des pluies, l'embroussaillement est contrôlé avec un feu de pleine saison sèche tous les 3 ans et il est réduit de moitié en 10 ans, si le feu est appliqué tous les 3 ans, mais seulement lorsque les pluies sont installées (après $100 \mathrm{~mm}$ de pluie).

L'appoint d'une prairie cultivée a également été testé : au Nigeria, la chute de poids de saison sèche est remplacée par un gain de $10 \mathrm{~kg} /$ tête, lorsque les animaux pâturent en saison sèche, avec une charge d'l ha/tête, une superficie comprenant $2 / 3$ de savane et $1 / 3$ de Stylosanthes guyanensis. Sur pâture de Stylo, un gain de $45 \mathrm{~kg} / \mathrm{ha}(45 \mathrm{~kg} /$ tête) est obtenu sur les 6 mois de saison sèche, avec une charge de 1 ha/tête. Avec exploitation toute l'année, en rotation avec une charge de $1,25 \mathrm{ha} / \mathrm{tête}$, le gain de poids vif est de $135 \mathrm{~kg} / \mathrm{ha}$ ( $168 \mathrm{~kg} / \mathrm{tête})$. Des résultats voisins ont été obtenus en limite nord du secteur périforestier à Bouaké (CHARRAY, 1973) où avec une charge de 0,55 ha/tête, un gain de poids vif de $175 \mathrm{~kg} / \mathrm{ha}(97 \mathrm{~kg} /$ tête) a été obtenu pendant les 5 mois de saison des pluies.

\section{BESOINS EN RECHERCHES COMPLÉMENTAIRES}

En définitive, les pâturages naturels potentiels de la zone tropicale humide sont constitués par toutes les formations végétales présentant un tapis graminéen appréciable, ainsi que tous les stades de jachères après abandon des cultures, dans la mesure où subsiste toute l'année des possibilités d'abreuvement.

Cependant parmi les graminées présentes, certaines sont appréciées par le bétail, d'autres sont consommées sans empressement et certaines sont délaissées. La qualité du parcours en résulte et, sous pâture, un déséquilibre s installe entre ces diverses espèces. Ce déséquilibre va s'accroissant avec la charge, la fréquence du broutage et les espèces les plus recherchées s'affaiblissent puis disparaissent.

Par ailleurs, l'importance du couvert ligneux entraîne, outre le risque accru de trypanoso miases, une modification de la flore herbacée. Les graminées recherchant l'ensoleillement sont éliminées dans les plages d'ombre au profit de graminées souvent moins productives ou d'espèces herbacées et subligneuses non pâturables.

L'estimation des ressources pastorales d'une région doit comprendre l'inventaire des pâturages potentiels qui sont en perpétuel remaniement, soit par la culture, soit par la pâture elle-même, car la production des parcours varie avec l'embroussaillement, l'ombre portée des ligneux alors qu'a priori elle ne dépend que du potentiel de fertilité lié aux types de sol et aux conditions hydriques des diverses situations topographiques.

Dans toute cette zone, les parcours potentiels et les parcelles cultivées sont étroitement imbriqués à l'exception de quelques territoires délaissés soit parce qu'ils constituaient des " marches" entre 2 ethnies rivales, soit parce que les conditions naturelles étaient impropres à l'établissement permanent de groupements humains (absence d'approvisionnement en eau, pauvreté des sols, présence d'endémies). Mais ces espaces libres sont peu à peu colonisés par l'homme au fur et à mesure que des techniques nouvelles permettent d'y rendre viables les conditions d'existence.

L'inventaire des ressources pastorales de cette zone se rapproche donc plutôt d'une étude cadastrale du parcellaire des terroirs villageois avec des moyens d'investigation actualisés (photos aériennes) et une cartographie à grande échelle, la classification des parcours répertoriés étant basée d'une part sur 
les conditions édaphiques et d'autre part sur l'importance de l'embroussaillement, la nature des ligneux (rejets, gaulis, perchis, futaie) et du degré de recouvrement des couronnes:

- savane herbeuse (grassland) ( $\left.{ }^{*}\right)$ pour un couvert inférieur à 2 p. 100 ,

- savane arborée (wooded grassland) pour un couvert inférieur à 20 p. 100 ,

- savane arbustive (bushland) pour un couvert inférieur à 40 p. 100 ayec des arbustes à troncs peu différenciés et de taille inférieure à $5 \mathrm{~m}$,

- savane boisée (grassed woodland) pour un couvert inférieur à 40 p. 100 avec des arbres à troncs bien différenciés et de taille supérieure à $5 \mathrm{~m}$,

- forêt claire (woodland) pour un couvert supérieur à 40 p. 100.

Dans un premier stade, l'inventaire des parcours potentiels pourra être illustré par un calque équipant les cartes topographiques à $1 / 200000$ (type I. G. N.) où figurent déjà le relief, l'hydrographie, les voies de communication, les agglomérations.

L'amélioration de la gestion des parcours nécessite des investissements fonciers à réaliser par les exploitants des parcours, ce qui suppose qu'au préalable, un statut juridique des parcours soit élaboré, constituant une sorte de code rural en vue d'assurer à l'utilisateur une pérennité d'usage afin de bénéficier des fruits de l'amélioration effectuée, sans pour autant instituer un droit de propriété inaliénable et transmissible aux héritiers.

Ce préalable étant respecté, les connaissances fragmentaires déjà acquises permettraient de vulgariser des principes élémentaires de gestion des parcours :

- attribution, bornage et si possible clôture d'unités-parcours gérées par un exploitant (individu, famille, coopératives...) ;

- découpage de l'unité-parcours en 2 blocs afin de pratiquer une rotation primitive en balancier respectant rigoureusement un repos de 3 à 4 semaines (le temps de séjour serait donc de même durée, car il est utopique et non économique d'envisager le passage immédiat

(*) Les termes entre parenthèses sont des termes usuels anglophones. d'un élevage de cueillette en vaine pâture à un élevage en rotation complexe respectant un temps de séjour de 2 à 3 j);

- protection de l'unité-parcours contre les feux courants par des pare-feux extérieurs établis par feux très précoces sur terrains cuirassés, par brûlis précoces de bandes de végétation herbacée pailleuse préalablement couchée et brisée par le traînage de grosses branches ou d'un traîneau de poutrelles, ou par bandes de cultures annuelles précoces (mil, arachide...);

- lutte contre l'embroussaillement en ne brûlant que tous les 2 ou 3 ans après l'établissement des pluies, les plages de refus pailleux à l'intérieur des blocs ;

- respect d'une charge en bétail d'l ha par $100 \mathrm{~kg}$ de poids vif en secteur soudano-guinéen à 0,75 ha par $100 \mathrm{~kg}$ de poids vif en secteur périforestier, une supplémentation équivalent à $1 \mathrm{~kg}$ de graines de coton par tête étant indispensable pendant les 2 derniers mois de saison sèche.

A la vulgarisation de ces principes de gestion de parcours de première génération, devrait être associée une expérimentation d'accompagnement destinée à préciser les connaissances d'une part sur la production secondaire des parcours en gain de poids vif par des troupeaux de taurillons avec pesées mensuelles et d'autre part sur la surveillance continue des parcours en contrôlant par inventaire statistique l'évolution de la composition du tapis graminéen de chaque type de parcours ainsi que celle du recouvrement des strates ligneuses. Sans compliquer la rotation des parcours, 2 à 3 charges seraient comparées par rotation sur 2 blocs pour des unités expérimentales englobant les principaux types de parcours du secteur. La charge moyenne de début d'expérimentation serait définie par l'estimation de la biomasse appétible en fin de période active. Les résultats déjà acquis confirment d'ailleurs que cette estimation est raisonnable : 2 ha/UBT à Abokouamekro (C. I.) pour une estimation de $130 \mathrm{j} /$ ha de pâture; 1,5 ha/UBT à Wakwa (plateaux du Cameroun) pour $210 \mathrm{j} / \mathrm{ha}$ de pâture. Les charges comparées seraient à \pm 30 p. 100 de la charge moyenne, par exemple 90,125 et $160 \mathrm{~kg} / \mathrm{ha}$ pour Abokouamekro.

Le troupeau élémentaire de l'expérimentation devrait être d'environ 30 taurillons en bon état sanitaire et âgés de 15 mois à 2 ans dont 
l'évolution du poids moyen, si l'expérimentatlon est satisfaisante, devrait :

- accuser une croissance compensatrice de 2 à 3 semaines en début des pluies;

- progresser d'environ $150 \mathrm{~g} / \mathrm{j} / 100 \mathrm{~kg}$ de poids vif pendant la période active de végétation ;

- stagner pendant les 3 premiers mois de saison sèche ;

- ensuite décroître d'environ $150 \mathrm{~g} / \mathrm{j} / 100 \mathrm{~kg}$ de poids vif jusqu'aux pluies.

De meilleures performances feraient supposer une charge favorable alors que des performances inférieures pourraient provenir d'une charge inadaptée. Une supplémentation serait alors distribuée pour sauvegarder le capital cheptel. L'expérimentation serait poursuivie pendant 2 ans avec le même troupeau, les effets de la charge étant par ailleurs contrôlés sur le parcours par l'estimation des refus, les variations de la composition floristique et surtout le comportement des espèces les mieux appétées. En cas de modification dangereuse du couvert végétal, après 2 ans ou multiple de 2 ans, le bloc expérimental pourrait être mis hors pâture, protégé des feux courants puis brûlé après l'établissement des pluies.

Des essais de restauration de parcours doivent aussi être poursuivis sur pâturages déjà dégradés, en particulier par surcharge en pâture continue, soit par travail du sol uniquement, soit par travail du sol et implantation de graminées à stolons (Cynodon plectostachyus, Digitaria umfolozi...) ou des graminées rustiques peu exigeantes (Melinis minutiflora).

Des essais d'amélioration des parcours doivent être conduits également, de préférence sur les sols à fort potentiel de fertilité en vue d'une intensification future de l'élevage. Ces essais doivent être amorcés en réseau multilocal avec test d'introduction et de production d'espèces locales en culture pure ou association graminéeslégumineuses, mais aussi d'espèces exotiques (collections australiennes par exemple).

\title{
SUMMARY
}

\author{
Grasslands in the humid tropics. \\ Present knowledge and further research requirements
}

In the humid tropics, basic prunciples of grassland management can be formulated from the knowledge of vegetation formations and their net primary productivity. Yet the grazed ecosystems can be conceived only by integrating the grass-eating animal into it and it is urgent to specify their secondary production as meat for the data obtained from the farms are often inadequate and too fragmentary.

\section{RESUMEN}

Pastos de la zona tropical húmeda

Conocimientos adquiridos $y$ necesidades de investigaciones complementarias

En zona tropical húmeda, los buscadores especializados en el estudıo de los pastos naturales ya han adquirido conocimientos permitiendo elaborar algunos principios fundamentales de gestión de los pasturajes (range management) susceptitles de ser utslizados como temas de base para la vulgarızación. Dichos principios son el respeto del tiempo de descanso, durante tres semanas a un mes, de los rebrotes de las gramíneas y, para limitar la invasión de malezas, la necesidad de un fuego al fin de temporada seca, cada tres años, con una provisión de pajas proviniendo de un no pastoreo durante un año.

Sin embargo, al inventario de los tipos de pasturaje, a la estimación de la producción por cortas y pesadas, a la elaboración de series evolutivas por observación minuciosa de los pasturajes explotados para la ganaderia tradicional o mejorada, se necesıta añadı ımperativamente una multiplicación de ensayos de cargo con el fin de testar mediante un rebaño homogéneo la producción secundarıa (evaluada en $\mathrm{kg}$ de peso vivo por hectárea) susceptible de ser obtenida con varias cargas y varios esquemas de gestión de los pasturajes.

Paralelamente, se necesita efectuar en red multilocal ensayos de restauración de los pasturajes y de mejora de la producción de los pasturajes con enriquecimiento de la flora, por ejemplo. 


\section{BIBLIOGRAPHIE}

1. BOUDET (G.). Manuel sur les pâturages tropicaux et les cultures fourragères, $2^{e}$ éd. Paris, MaisonsAlfort, Ministère de la Coopération, I. E. M. V. T., 1975,235 p.

2. BOUDET (G.).Problèmes posés par l'estimation de la capacité de charge d'un pâturage naturel tropical. Addis-Abéba, C. I. P. E. A.-I. L. C. A. ; Actes du Colloque sur l'inventaire et la cartographie des pâturages tropicaux africains, Bamako, Mal1, 3-8 mars 1975, pp. 265-267.

3. CHARRAY (J.). Embouche de zébus peuls sur Stylosanthes gracilis I. E. M. V. T., Colloque de Dakar sur l'embouche intensive des bovins en pays tropicaux, 4-8 décembre 1973, pp. 167-170.
4. C. S. A. Phytogéographie (Yangambi 1956). Londres, C. C. T. A., 1956 (publ, $n^{0} 22$ ), 35 p.

5. DE LEEUW (P. N.), BRINCKMAN (W. L.). Pasture and rangeland improvement in the northern Guinea and Sudan zone of Nigeria. Ibadan, International symposium on animal production in the tropics, 1973, $13 \mathrm{p}$.

6. PIOT (J.), RIPPSTEIN (G.). Rapports annuels d'activités de la station fourragère de Wakwa N'Gaoundéré (Cameroun), 1964 à 1975.

7. PRATT (D. J.), GREENWAY (P. J.), GWYNNE (M. D.). Classification of East African rangeland with an appendix on terminology. J. appl. Ecol., 1966 (3) : 369-382. 